Michenthaler, Johanna; Laufenberg-Beermann, Anne von \title{
Hauswirtschaftliche (Berufs-)Bildung braucht Orientierung am nachhaltigen
} Mindset

Haushalt in Bildung \& Forschung 8 (2019) 2, S. 107-119

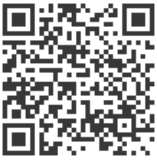

Quellenangabe/ Reference:

Michenthaler, Johanna; Laufenberg-Beermann, Anne von: Hauswirtschaftliche (Berufs-)Bildung braucht Orientierung am nachhaltigen Mindset - In: Haushalt in Bildung \& Forschung 8 (2019) 2, S. 107-119 URN: urn:nbn:de:0111-pedocs-221312 - DOI: 10.25656/01:22131

https://nbn-resolving.org/urn:nbn:de:0111-pedocs-221312

https://doi.org/10.25656/01:22131

in Kooperation mit / in cooperation with:

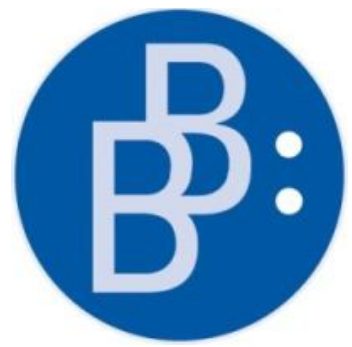

https://www.budrich.de

\section{Nutzungsbedingungen}

Dieses Dokument steht unter folgender Creative Commons-Lizenz: http://creativecommons.org/licenses/by-sa/4.0/deed.de - Sie dürfen das Werk bzw. den Inhalt vervielfältigen, verbreiten und öffentlich zugänglich machen sowie Abwandlungen und Bearbeitungen des Werkes bzw. Inhaltes anfertigen, solange sie den Namen des Autors/Rechteinhabers in der von ihm festgelegten Weise nennen und die daraufhin neu entstandenen Werke bzw. Inhalte nur unter Verwendung von Lizenzbedingungen weitergeben, die mit denen dieses Lizenzvertrags identisch, vergleichbar oder kompatibel sind.

Mit der Verwendung dieses Dokuments erkennen Sie die Nutzungsbedingungen an.

\section{Terms of use}

This document is published under following Creative Commons-License: http://creativecommons.org/licenses/by-sa/4.0/deed.en - You may copy distribute and transmit, adapt or exhibit the work or its contents in public and alter, transform, or change this work as long as you attribute the work in the manner specified by the author or licensor. New resulting works or contents must be distributed pursuant to this license or an identical or comparable license.

By using this particular document, you accept the above-stated conditions of use.

\section{Kontakt / Contact:}

peDOCS

DIPF | Leibniz-Institut für Bildungsforschung und Bildungsinformation Informationszentrum (IZ) Bildung

E-Mail: pedocs@dipf.de

Internet: www.pedocs.de

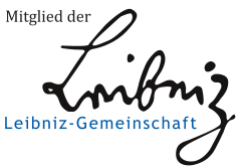




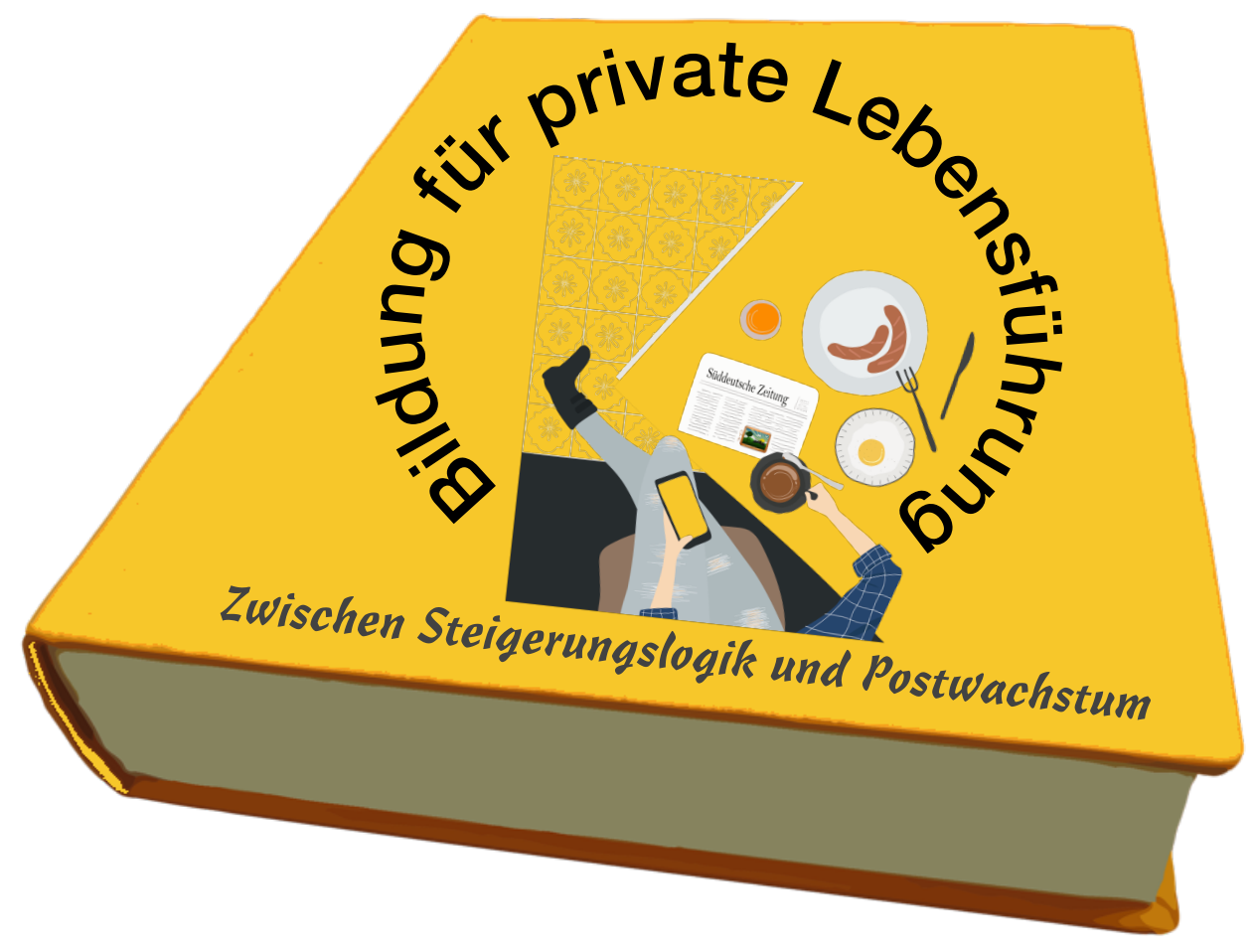


Kirsten Schlegel-Matthies

Editorial 2

Katharina Witterhold

Konsum als politische Praxis? Transformation des Alltags

als Herausforderung und Chance 3

Kora Kristof

Erfolgsbedingungen für gesellschaftliche Veränderungsprozesse

Kirsti Dautzenberg

Marktwächter Digitale Welt: Schöne neue Shopping-Welt?

Angela Häußler

Who cares? Sorgearbeit als individuelle Aufgabe

und gesellschaftliche Herausforderung

Werner Brandl

Conduct of everyday life - some views and insights 54

Claus Tully

Jugend - Konsum - Digitalisierung: Aufwachsen in digitalen Welten

Kirsten Schlegel-Matthies

Haushaltsbezogene Bildung - quo vadis?

Daseinsvorsorge und Lebensführung im Wandel 88

Johanna Michenthaler \& Anne von Laufenberg-Beermann

Hauswirtschaftliche (Berufs-)Bildung

braucht Orientierung am nachhaltigen Mindset

,Rezension“

Schöler, H. (2019). Entwicklung und Bildung im Kindesalter.

Eine Kritik pädagogischer Begriffe und Konzepte 
Johanna Michenthaler \& Anne von Laufenberg-Beermann

\section{Hauswirtschaftliche (Berufs-)Bildung braucht Orientierung am nachhaltigen Mindset}

Mit dem ProfESus-Konzept ist es gelungen, aktuelle pädagogische Ansätze mit den Forderungen der Nachhaltigkeitsbildung zu verknüpfen und Unterrichtskonzepte erfolgreich zu implementieren. Lehrkräfte der hauswirtschaftlichen Berufsbildung erhalten konkrete Hinweise für die innovative Gestaltung von Unterricht. Damit können diese einen wesentlichen Beitrag für die Zukunftsfähigkeit der Hauswirtschaft leisten.

Schlüsselwörter: Bildung für Nachhaltige Entwicklung (BNE), Blended Learning-Kurs, Nachhaltiges Mindset, Lehrerbildung, Evaluierung, ProfESus

With the ProfESus concept, it has been possible to link current pedagogical approaches with the demands of sustainability education and to successfully implement teaching concepts. Teachers of home economics vocational training receive concrete tips for the innovative design of teaching. In this way, they can make a significant contribution to the future viability of home economics.

Keywords: Education for Sustainable Development (ESD), blended learning course, sustainable mindset, teacher training, evaluation, ProfESus

\section{Gesellschaftspolitische Forderungen an die Berufsbildung}

Laut UNESCO (2015) sind folgende Schlüsselaspekte für eine innovative Bildung hervorzuheben: „Die Welt verändert sich - auch die Bildung muss sich ändern!“ Dabei müssen wir die Bildung in einer sich verändernden Welt neu durchleuchten (vgl. UNESCO, 2015, S.4 ff.).

Bildung für nachhaltige Entwicklung (BNE) fordert die Lerner-zentrierte Gestaltung von Unterricht, sowie vielfältige Lernumgebungen - physisch, online und virtuell -, welche Lernende inspirieren im Sinne der Nachhaltigkeit zu handeln. Durch Interaktion soll exploratives, handlungsorientiertes und transformatives Lernen ermöglicht werden.

Durch die Orientierung an Lernergebnissen und die Stimulierung des Lernens müssen Kernkompetenzen wie kritisches und systemisches Denken, kooperative Entscheidungsfindung und Übernahme von Verantwortung für gegenwärtige und zukünftige Generationen gefördert werden. 


\section{Hauswirtschaftliche (Berufs-)Bildung}

Die Bildung für nachhaltigen Konsum zielt darauf ab, Wissen, Werte und Fähigkeiten zu vermitteln, die es Konsumenten generell, aber auch Fachkräften in der Hauswirtschaft ermöglichen, zu Akteuren des Wandels hin zu einem nachhaltigeren Konsumverhalten zu werden. Sie integriert die Grundrechte und -freiheiten, einschließlich der Verbraucherrechte, und zielt darauf ab, die Bürger in die Lage zu versetzen, sich in informierter und ethischer Weise an der öffentlichen Debatte und Wirtschaft zu beteiligen (vgl. UNEP, 2010).

Forschungsergebnisse haben gezeigt, dass in privaten Haushalten, Großküchen und gastorientierten Betrieben oftmals nicht nur eine große Notwendigkeit an verbesserten Umweltmaßnahmen, sondern auch ein großes Potenzial für die Umsetzung nachhaltigen Handelns zum Schutz der Umwelt besteht. Diese Berufsfelder haben auch einen großen Einfluss auf die sozialen und wirtschaftlichen Verbesserungen für Arbeitskräfte weltweit.

\section{Intention des ProfESus-Projektes}

Das europäische Projekt „Fokus auf Nachhaltigkeit - Bildung für Fachkräfte in haushalts- und gastorientierten Betrieben" (ProfESus) zielte darauf ab, eine innovative Blended-Learning-Lehrerweiterbildung zu entwickeln, welche das systematische nachhaltige berufliche Handeln in hauswirtschaftlichen Betrieben fördert.

Das Projektteam, bestehend aus sechs europäischen Partnern, reflektierte aktuelle Anforderungen, pädagogische Theorien, Methoden, Werkzeuge und Konzepte für eine zukunftsorientierte Lehrerweiterbildung im Bereich der beruflichen Bildung.

Basierend auf den Forderungen der UNESCO (2015) sollte das Projekt einen Beitrag zur Verbesserung der Qualität der Berufsbildung leisten. Es braucht einen stärkeren Fokus auf Lehrkräfte als „Change Agents“ in allen Bereichen.

Laut der Wirtschaftskommission der Vereinten Nationen für Europa stellen die Kompetenzen der Lehrkräfte häufig den Engpass bei der Qualitätsverbesserung der Bildung dar (vgl. UNECE, 2011). Das Umweltprogramm der Vereinten Nationen erklärte, dass daher die Neuausrichtung der Lehrerbildung in Bezug auf Nachhaltigkeit eine große Herausforderung für Bildungseinrichtungen bedeutet (vgl. UNEP, 2010).

Basierend auf dem neuen Ansatz von Bildung sowie deren Bedeutung und der Rolle von Lehrkräften muss geklärt werden, wie Bildung entwickelt werden soll und muss, um das mächtige Instrument für die Transformation zu sein.

Mit dem Fokus auf Bildung für Nachhaltige Entwicklung (BNE) wurde der Blended-Learning-Lehrgang „Discovering a sustainable Mindset“ installiert. Hier wird Lehrkräften ermöglicht, selbst ein Mindset für Nachhaltigkeit zu entwickeln, sowie innovative Bildungsansätze und -methoden anzuwenden, um die Entwicklung eines nachhaltigen Mindsets bei Lernenden der hauswirtschaftlichen Berufsbildung zu fördern. 
Ein Mindset für Nachhaltigkeit beinhaltet demnach nicht nur Wissen, Fertigkeiten und Fähigkeiten, es umfasst auch Werteorientierungen und daraus resultierendes Verhalten. Ziel ist es, Veränderungs- und Transformationsprozesse anzustoßen, ähnlich den Ergebnissen, die Stanszus et al. (vgl. 2017, S.7 f.) postulieren, um Routinen zu durchbrechen, die Einstellungs-Verhaltens-Lücke zu verringern, kongruente Entscheidungen zu treffen, die auf nichtmaterielle Werte fokussieren und um pro-soziale sowie pro-ökologische Verhaltensmuster durch Empathie und Zusammenarbeit zu fördern.

Die Ergebnisse des Projekts zeigen praxisnah, dass Lehrkräfte mittels des ProfESus-Pathway pädagogische Kompetenzen zeitgleich mit den Nachhaltigkeitskompetenzen entwickeln können (vgl. UNECE, 2011). Weiter wurden Lernsettings, in denen sich Aspekte innovativer Bildung in Lernhandlungen widerspiegelten, im Sinne der BNE für Lernende in der hauswirtschaftlichen Berufsbildung interkontinental geplant und umgesetzt. Der ProfESus-Lehrgang soll zu einer routinierten fachdidaktischen Planung beitragen, um das Potenzial einer nachhaltigen Berufsbildung zu erweitern. Die Umsetzung des ProfESus-Projekts wurde durch das Erasmus+Programm der Europäischen Union finanziell gefördert.

\section{Kompetenzorientierung in der hauswirtschaftlichen Berufsbildung}

In der europäischen Berufsbildung mit dem europäischen Qualifikationsrahmen (ec.europa.eu/eqf) steht der Erwerb von Kompetenzen im Vordergrund. Berufliche Kompetenz geht einher mit beruflichen Fähigkeiten, welche mehr beschreiben als nur Wissen (ebd.). Die individuelle Kompetenz beinhaltet ein Netzwerk von verwandten Aspekten wie Wissen, Fähigkeiten und Fertigkeiten, Verständnis, Handlung, Erfahrung und Motivation (vgl. Weinert 2001). Eine nachhaltiges Mindset ist Voraussetzung, um spezifische Problemsituationen im Arbeitsleben im Hinblick auf Nachhaltigkeit zu bewältigen. Durch den Einsatz von Mindset-orientiertem Unterricht sollen die Schülerinnen und Schüler lernen, wie sie (träge) Kenntnisse in professionelles Handeln umsetzen und das vorhandene Wissen bei berufsbezogenen Aktivitäten nutzen können. Diese Gesamtfähigkeiten gelten als Maßstab für den Erfolg des Lernprozesses in der beruflichen Bildung für nachhaltige Entwicklung.

\section{Methoden des ProfESus-Projektes}

Erfahrene Lehrkräfte, Pädagoginnen und Pädagogen haben im Projektzeitraum 2016 bis 2018 ein innovatives Konzept für Lehrkräfte in der hauswirtschaftlichen Berufsbildung erarbeitet, um eine nachhaltige Entwicklung zu fördern. Dieses Konzept wurde in einer Blended-Learning-Lehrerweiterbildung (8 ECVETS/ECTS) umge- 


\section{Hauswirtschaftliche (Berufs-)Bildung}

setzt. Es berücksichtigt die obengenannten gesellschaftspolitischen und pädagogischen Anforderungen. An dem ersten internationalen Pilotlehrgang nahmen Lehrkräfte, Ausbilderinnen und Ausbilder der Berufsbildung ( $\mathrm{n}=43$ ) aus 15 verschiedenen Ländern teil. Im Rahmen der ersten Projektphase wurden folgende Forschungsfragen untersucht:

- Wie soll Bildung für Hauswirtschaft aussehen, damit Lehrkräfte und Lernende ein Mindset für Nachhaltigkeit entwickeln?

- Welche Aspekte müssen berücksichtigt werden, um innovativen Unterricht für Nachhaltigkeit in hauswirtschaftlichen Betrieben zu erreichen?

- Wie plant man innovativen Unterricht für Nachhaltigkeit?

- Wie evaluiert man Unterricht für Nachhaltigkeit bzw. das wachsende Mindset in Lernenden?

- Welche Aspekte sollten bei der Planung und Evaluation eines Lehrerweiterbildungslehrgangs zur nachhaltigen hauswirtschaftlichen Bildung beachtet werden?

In der praktischen Pilotphase wurden die entwickelten Lehrgangsinhalte und -konzepte einschließlich Methoden und Aufgabenstellungen in vier verschiedenen (Online- und Präsenz-)Modulen umgesetzt und evaluiert. Ziel dieser Pilotphase war es, Feedback von den teilnehmenden Lehrkräften zum ProfESus-Lehrgang zu erhalten, um den oben genannten Anforderungen an eine Berufsbildung für Nachhaltigkeit in hohem Maße gerecht zu werden. Nach dem Pilotlauf des Lehrganges wurden die Evaluierungsergebnisse genutzt, um das Lehrgangskonzept bis hin zu Materialien und Aufgabenstellungen zu optimieren.

Die Datengenerierung erfolgte dabei über Online-Fragebögen und Feedbackgespräche in Präsenzveranstaltungen.

Die Best-Practice-Lernaktivitäten wurden mittels einer Vorlage für Unterrichtsplanung und einer Checkliste bewertet, die während der Projektarbeit mit Unterstützung von Experten und Multiplikatoren erstellt wurden.

Die Evaluierung des wachsenden Mindsets für Nachhaltigkeit von hauswirtschaftlichen Lehrkräften wurde mittels qualitativer Analyse der Lerntagebücher durchgeführt. Unter Einsatz der Leximancer-Software wurde die qualitative Analyse hinsichtlich differenzierter Fragestellungen durchgeführt und ausgewertet. Die Ergebnisse dienen einerseits als Indikatoren für die Entwicklung eines Mindsets für Nachhaltigkeit und andererseits als Gradmesser für den Erfolg des ProfESusLehrganges.

Der basierend auf den Evaluierungsergebnissen verbesserte ProfESus-Lehrgang einschließlich aller Unterrichtsmaterialien und die Best-PracticeUnterrichtsplanungen sind als Open-Source-Quellen unter www.profesus.eu veröffentlicht. 


\section{Hauswirtschaftliche (Berufs-)Bildung}

\section{Resultate}

Die Implementierung von innovativem Unterricht zur Nachhaltigkeit in der Hauswirtschaft ist bei bestehender Bereitschaft von Lehrkräften und Institutionen möglich, sofern oben genannte Anforderungen umgesetzt werden. Dabei können entsprechende Lehrgänge in der Weiterbildung von Lehrkräften eine effiziente Alternative darstellen, wie die Ergebnisse des ProfESus-Lehrganges zeigen.

\section{Wie soll Bildung für Hauswirtschaft aussehen, damit Lehrkräfte und Lernende ein Mindset für Nachhaltigkeit entwickeln?}

Das ProfESus-Projekt stellte verschiedene pädagogische Theorien und Lehransätze gegenüber, die innovative Bildung und Bildung für eine nachhaltige Entwicklung unterstützen. Die folgenden Ergebnisse fassen die Anforderungen und Erwartungen an Bildung und Bildung für nachhaltigen Konsum zusammen:

Die Lernmethoden und pädagogische Ansätze müssen das Lernen als aktiven und konstruktiven Prozess gestalten. Dazu zählen beispielsweise situatives Lernen laut Lave (vgl. 1988) und innovative Formen des Lehrens, Lernens und Beurteilens wie Sharples et al. (vgl. 2015) postulieren.

Die aus Sicht von ProfESus am relevantesten für BNE sind:

- Grenzüberschreitendes Lernen

- Lernen durch Argumentation

- Technologien zur Unterstützung des Lernens

- kontextbasiertes Lernen

- computergestütztes Denken

- adaptives Lehren

- Analyse von Emotionen

- unsichtbares Beurteilen

- Provokation als Methode

- fürsorgliche Konfrontation

- kreativitätsfördernder Unterricht

- Fallstudien im aktiven Unterricht

- Szenarien in Bildung und Lehre

- Storytelling

Unter Berücksichtigung der Ansätze der Bildung für nachhaltige Entwicklung wurde an der Hochschule für Agrar- und Umweltpädagogik Wien das Konzept der Grünen Pädagogik (vgl. Hochschule, 2018) erarbeitet, welches im Zuge des ProfESusProjekts durch Integration der UNECE-Kompetenzen für Nachhaltigkeit zum ProfESus-Pathway weiterentwickelt wurde. Dieses Konzept des „ProfESus Pathway to Discovering a Sustainable Mindset" ist ein Leitfaden zur schrittweisen Planung in- 
novativer Lernprozesse, damit Lernende/Fachkräfte ihr nachhaltiges Mindset entwickeln können (siehe Abbildung 1).

Sichtbar werden im ProfESus Pathway auch die sechs Schlüsselkompetenzen für nachhaltiges Handeln: Zusammenarbeit, systemisches Denken, kritisches Denken, strategisches Denken, werteorientiertes Denken und zukunftsorientiertes Denken.

Blockzitate

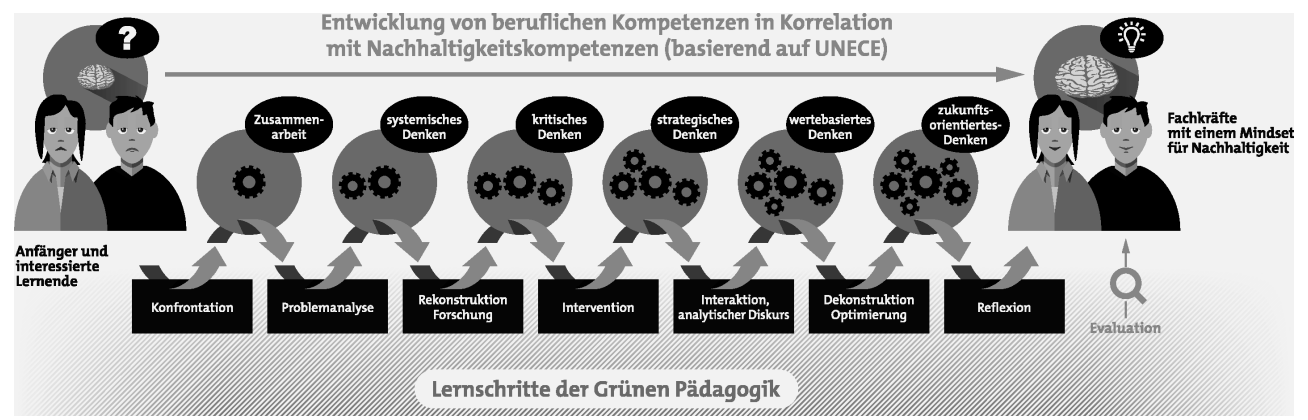

Abb. 1: ProfESus-Pathway zur Entwicklung eines persönlichen Mindset für Nachhaltigkeit (Quelle: ProfESus Newsletter|AUGUST 2018, S. 6)

Der ProfESus-Pathway beschreibt und symbolisiert einerseits den Kontext, den Lehrkräfte/Pädagogen berücksichtigen sollten, und andererseits den Prozess, den Lehrerkräfte/Pädagogen im Detail planen sollten, um die Lernenden bei der Entdeckung ihrer nachhaltigen Denkweise zu unterstützen. Der innovative Ansatz des ProfESus-Konzepts integriert Fachkompetenzen basierend auf den jeweiligen Curricula und Nachhaltigkeitskompetenzen (modifiziert nach UNECE, 2011) für Lernende in der Berufsbildung.

Aus den Erkenntnissen des ProfESus-Projektes ist festzustellen, dass ein sich entwickelndes Mindset für Nachhaltigkeit nur dann gelingt, wenn der Lernprozess die Weiterentwicklung beruflicher Kompetenzen und eine fortschreitende Entwicklung der Nachhaltigkeitskompetenzen umfasst. Ein isolierter, einseitiger Ansatz der Entwicklung beruflicher Kompetenzen ohne die Einbindung der Nachhaltigkeitskompetenzen, führt nicht zu den notwendigen Verknüpfungen von Wissen, Fähigkeiten und Fertigkeiten, Wertehaltungen (siehe Abbildung 2). Daher ist die Anwendung von situativem Lernen, d.h. Lernen an Fallbeispielen, Szenarios oder am praktischen Beispiel sowie der Einsatz schüleraktivierender Methoden unerlässlich.

\section{Welche Aspekte müssen berücksichtigt werden, um innovativen Unterricht für Nachhaltigkeit in hauswirtschaftlichen Betrieben zu erreichen?}

Folgende Aspekte sollten für erfolgreiche berufliche Lernprozesse berücksichtigt werden: Es sollten Lerner-zentrierte Methoden eingesetzt werden, die Lernende 
durch reflektierende Provokation, persönliche Betroffenheit, Irritation (um außerhalb der Norm zu denken), Bewusstseinsbildung und der Motivation zur nachhaltigen Entwicklung aktivieren.

Lehrkräfte der Berufsbildung sollten dabei verschiedene Methoden und Werkzeuge einsetzen und das Lernen vor Ort durch betriebliche Fallbeispiele, Szenarien und Geschichten unterstützen. Widersprüche und Diskrepanzen sollten in den aktiven Lernprozess einbezogen werden.

In der schulischen Berufsausbildung besteht ein großer Bedarf des Lernens in verschiedenen Lernumgebungen (z.B. Schulklassen, Technikräume, Betriebe, Natur, Museen, Institute), um Inspiration und Reflexion zu fördern.

\section{Wie plant man innovativen Unterricht für Nachhaltigkeit?}

Lehrkräfte der Berufsbildung benötigen Unterstützung und Anleitung bei der Planung von innovativen Unterrichtsprozessen, welche die Nachhaltigkeit interdisziplinär berücksichtigt. Dies wurde im Rahmen des ProfESus-Lehrganges durch eine detaillierte Vorlage für die Planung von Lernaktivitäten (https://profesus.eu/fileadmin/user_upload/Outputs/German/ProfESus_Unterrichtspla n_de.docx) unterstützt.

\section{Wie evaluiert man Unterricht für Nachhaltigkeit bzw. das wachsende Mindset in Lernenden?}

Um den Prozess der Entwicklung eines nachhaltigen Mindset zu bewerten, müssen alle Dimensionen der Fach- und Nachhaltigkeitskompetenz berücksichtigt werden. Ein Mindset für Nachhaltigkeit ist auf folgende Kompetenzen - mit einem mehr oder weniger hohen Schwierigkeitsgrad - aufgebaut:

1. Wissen: (Fachkräfte wissen...)

2. Fähigkeiten und Fertigkeiten (Fachkräfte können...)

3. Teamwork: (Fachkräfte arbeiten mit anderen zusammen...)

4. Werte: (Fachkräfte sind Personen, die...)

In allen beruflichen Feldern bestimmen die Bildungsstufe und/oder die Tätigkeitsbeschreibung die Komplexität des jeweiligen beruflichen Aufgaben- und Verantwortungsbereichs. Zur Entwicklung eines persönlichen Mindset für Nachhaltigkeit müssen Fachkräfte Kompetenzen in allen vier o.g. Bereichen entwickeln, damit sie im Sinne der nachhaltigen Entwicklung ihre beruflichen Aufgaben planen, entscheiden, handeln, diskutieren und reflektieren können.

Die grafische Darstellung der ProfESus-Beurteilung (siehe Abbildung 2) zeigt den Kontext des Mindset für Nachhaltigkeit im beruflichen Handeln und gibt Hinweise, wie ein wachsendes Mindset für Nachhaltigkeit in Lern- oder Trainingsprozessen beurteilt werden kann. 


\section{Hauswirtschaftliche (Berufs-)Bildung}

Damit ein Mindset für Nachhaltigkeit bei Fachkräften und Lernenden beurteilt werden kann, müssen berufsbezogenen Aufgaben gestellt werden, so dass Lernende unter Beweis stellen können, dass sie fähig sind fach- und nachhaltigkeitsbezogen Wissen, Fertigkeiten und Werte einzusetzen, mit anderen im Team zusammenzuarbeiten, systemisch zu denken, kritisch zu denken, strategisch zu denken und zukunftsorientiert zu denken.

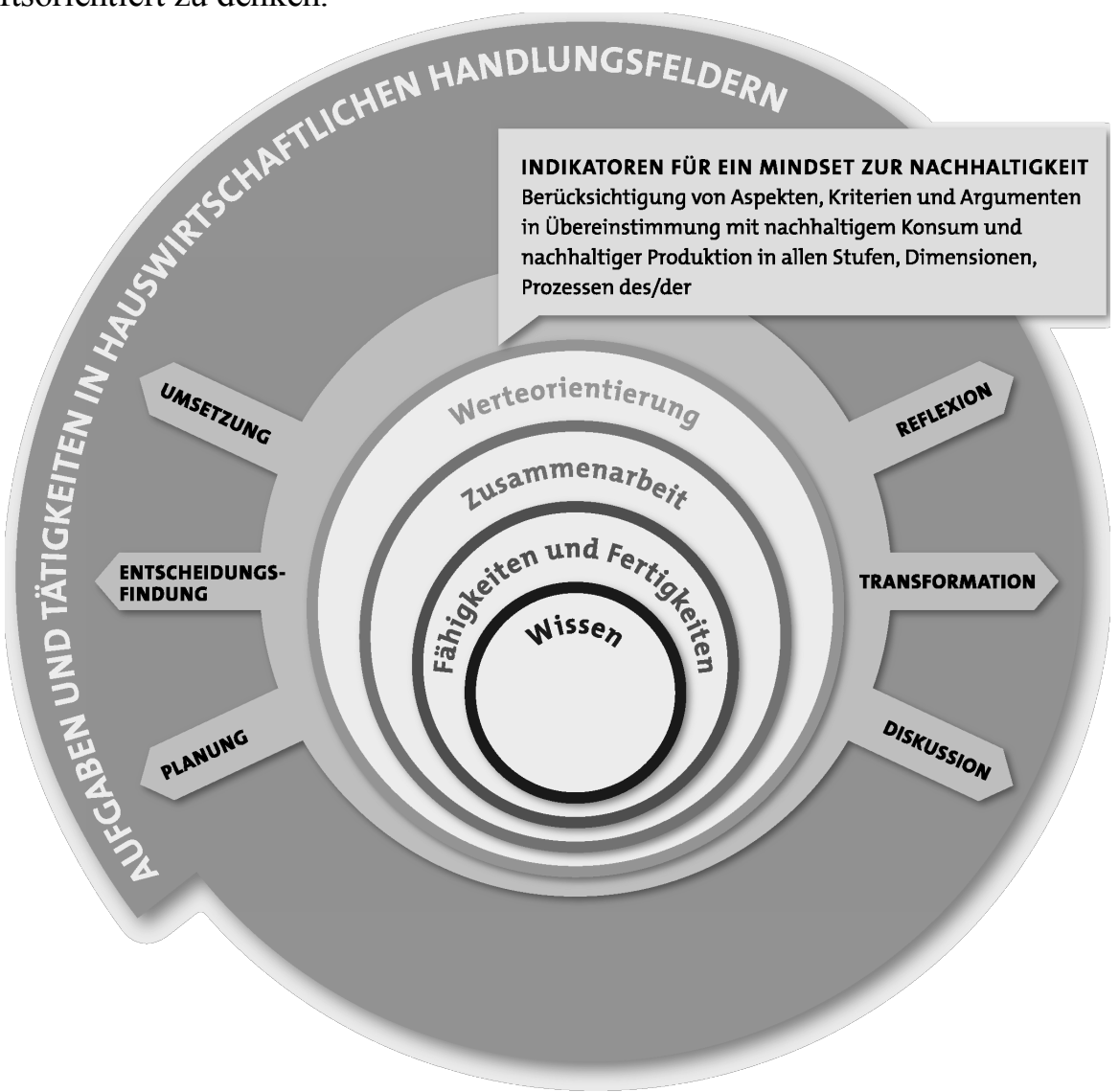

Abb. 2: ProfESus - Beurteilung des Mindset für Nachhaltigkeit (Quelle: ProfESus Newsletter | AUGUST 2018, S. 9)

Die ProfESus-Beurteilung (siehe Abbildung 2) leitet Lehrkräfte durch den Bewertungsprozess des kompetenzorientierten Lernens und hilft ihnen, eine Vorstellung davon zu bekommen, wie sie Kompetenzen und ein wachsendes Mindset in Lernenden und Fachkräften evaluieren können.

Auch in Beurteilungsprozessen ist die Verwendung von betrieblichen Fallbeispielen, Szenarien und Geschichten bzw. Problemstellungen im Zusammenhang mit den Berufsfeldern der Hauswirtschaft notwendig. 
Hauswirtschaftliche (Berufs-)Bildung |

\section{Welche Aspekte sollten bei der Planung und Evaluation eines Lehrerweiterbildungslehrgangs zur nachhaltigen hauswirtschaftlichen Bildung beachtet werden?}

Die aktuell empfohlenen pädagogischen und methodischen Ansätze für die Bildung für nachhaltige Entwicklung (BNE) sowie die von der UNECE definierten nachhaltigen Kompetenzen für Lehrkräfte wurden im innovativen Blended-Learning Lehrgang „ProfESus - Entwicklung eines Mindset für Nachhaltigkeit“ für zukünftige Fachkräfte in hauswirtschaftlichen Bereichen kombiniert. Das Curriculum für diese Lehrerweiterbildung wurde in vier Module strukturiert und mit 8 ECVETS/8 ECTS bewertet.

Das erste und vierte Modul des Lehrganges werden als fünftägige Präsenzphasen durchgeführt, in denen sich lernerzentrierte Methoden sowie innovative Formen des Unterrichts mit verschiedenen Methoden und Werkzeugen widerspiegeln. Das zweite und dritte Modul sind online in zwei Semestern zu absolvieren. Diese Module beinhalten neben einer Vielzahl von Aufgaben und digitalen Tools auch die Planung und Durchführung von Unterrichtseinheiten mit der eigenen Zielgruppe an Lernenden.

Modul 1 steht unter dem Titel „Startschuss zu einem nachhaltigen Mindset" und zielt darauf ab, das Bewusstsein der teilnehmenden Lehrkräfte für die Dimensionen und innovativen Ansätze von BNE einschließlich der Nachhaltigkeitskompetenzen von UNECE, welche in die berufliche Bildung integriert werden sollten, zu schärfen. Sie lernen den „ProfESus-Pathway zur Entwicklung des persönlichen Mindset für Nachhaltigkeit" als innovatives Unterrichtskonzept kennen.

Modul 2 „Bildung für Nachhaltigkeit“ konzentriert sich auf die Schlüsselkompetenzen für eine nachhaltige Entwicklung. Die Teilnehmenden lernen Theorien, Nachhaltigkeitskompetenzen und wertvollen Werkzeuge für Bildungsprozesse in BNE kennen. Das Modul ist in sieben Einheiten unterteilt, die sich befassen mit: Pädagogik für Nachhaltigkeit, Werkzeuge und Methoden für die Nachhaltigkeit, zukunftsorientiertes Denken, systemisches Denken, strategisches Denken, werteorientiertes Denken und Zusammenarbeit/Kooperation.

In Modul 3 unter dem Thema „Unterrichtsaktivitäten - Planung und Durchführung" lernen die Teilnehmenden die Phasen der Entwicklung eines Unterrichtsplans basierend auf dem Konzept des ProfESus-Pathway und den Vorlagen für Unterrichtsplanung kennen. Die Teilnehmenden planen, führen den Unterricht durch und evaluieren anschließend ihre Unterrichtsplanung.

Modul 4 mit dem Thema „Beurteilen von Lernprozessen und Entwickeln von Beiträgen für eine nachhaltige Zukunft" konzentriert sich auf die Bewertung der durchgeführten Unterrichtsplanungen und die Bewertung des entwickelten Mindset. Weiteres werden Strategien für die Transformation in Bildungssystemen und hauswirtschaftlichen Handlungsfeldern entwickelt.

Die folgende Abbildung 3 zeigt die wichtigsten Aspekte des Lehrganges: 


\section{Hauswirtschaftliche (Berufs-)Bildung}

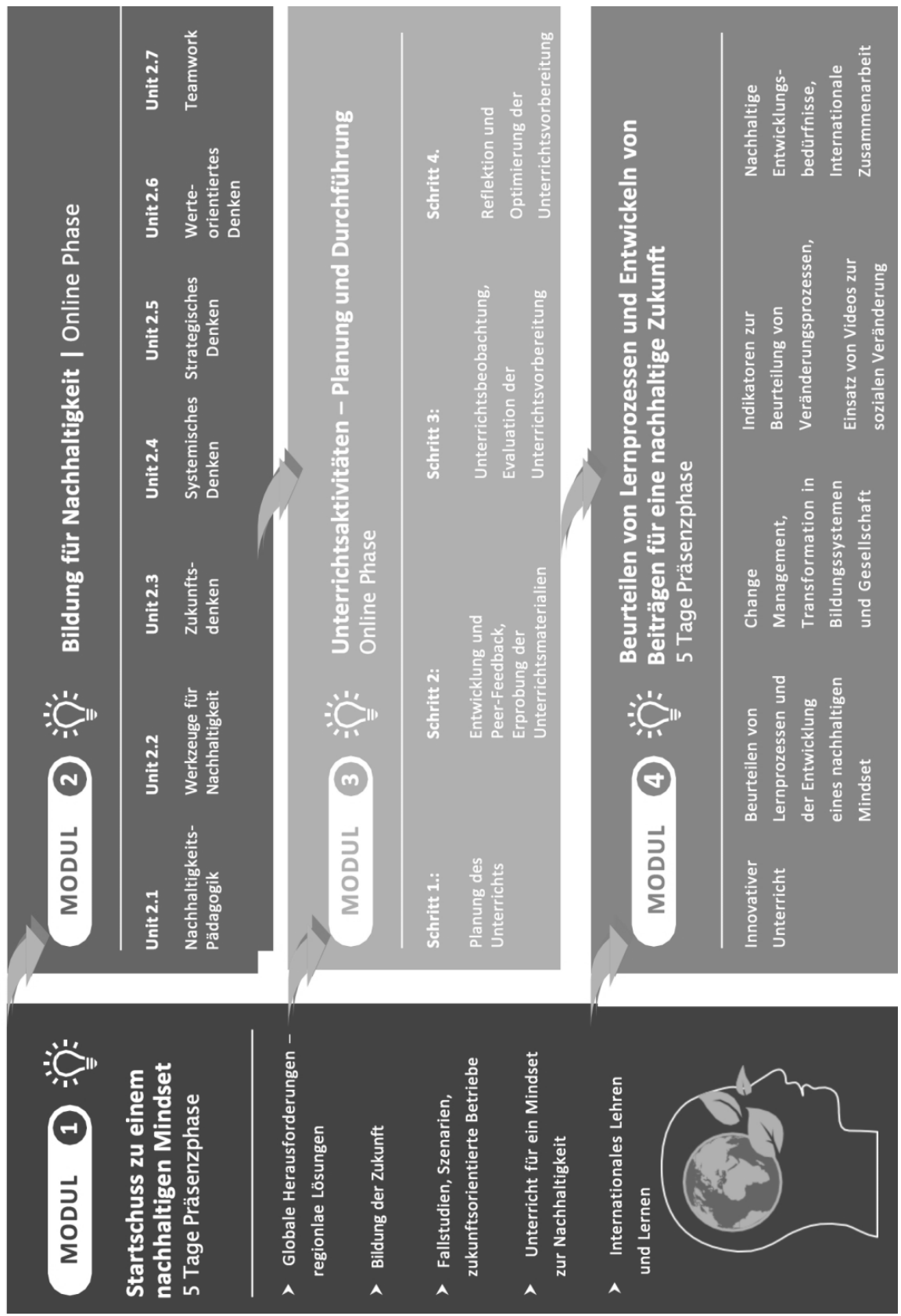

Abb. 3: Struktur des ProfESus Blended-Learning-Lehrgangs (Quelle: ProfESus Handbuch, S. 16) 
Als Online-Lernplattform wurde das Programm „Moodle“ eingesetzt, welches für die Durchführung der Online-Phasen des Lehrganges genutzt wurde, und darin stehen alle Lernmaterialien als Open Source unter folgendem Link zur Verfügung: https://profesus.eu/course/introduction-of-the-course/ bzw. https://profesus.eu/outputs/german/

\section{Qualität eines Lehrganges für Nachhaltigkeit}

Zur evidenzbasierten Evaluation des ProfESus-Lehrganges wurden folgende Instrumente und Tools angewendet:

- Entwicklung des internationalen Lehrganges begleitet von Expertenworkshops,

- Externer Qualitätsausschuss mit Expertinnen und Experten aus Bildung, Hauswirtschaft und Nachhaltigkeit evaluierten den ProfESus-Lehrgang vor dem ersten Pilotlauf und nach der abschließenden Überarbeitungsphase,

- Unterrichtsplanungen der teilnehmenden Lehrkräfte,

- Reflexion zu den Modulen des Lehrganges mittels Lerntagebuch einschließlich der qualitativen Analyse mittels Leximancer Software,

- Quantitative Befragungen hinsichtlich Inhalte, Methodik, Anforderungen, zeitlicher Umfang und der gewählten Schwerpunkte des Lehrganges,

- Selbstevaluation von Lehrgangs- und Modulleiterinnen und -leitern auf Basis der Lernergebnisse und Erfahrungen.

Eine ausführliche Beschreibung der Qualitätsaspekte sind im ProfESus Handbuch unter folgendem Link zu finden: https://profesus.eu/fileadmin/ user_upload/Outputs/English/ProfESus_Handbook_web.pdf

\section{Schlussfolgerungen für die Transformation der hauswirtschaftlichen (Berufs-) Bildung}

Die Lehreraus- und -weiterbildung in der Hauswirtschaft muss sich möglichst schnell innovativ verändern, damit Hauswirtschaft einen maßgeblichen Beitrag zur Transformation und Erreichung der Nachhaltigkeitsziele (vgl. United Nations, 2015) leisten kann.

Das Mindset zur Nachhaltigkeit in Lehrkräften und Ausbildern ist eine entscheidende Voraussetzung, um in den diversen Handlungsfeldern der Hauswirtschaft nachhaltige Bildung erfolgreich umzusetzen.

Berufsbildende Institutionen und deren Rahmenbedingungen müssen entsprechend den Anforderungen innovativer Bildung neu ausgerichtet und gestaltet werden, insbesondere Curricula, Lernumgebungen, Kooperationen, etc. 


\section{Hauswirtschaftliche (Berufs-)Bildung}

Monitoring und Unterrichtsevaluation sollten im Sinne der Entwicklung nachhaltiger Mindsets bei Lehrkräften und Lernenden neu aufgestellt werden.

\section{Diskussion und Ausblick}

Die Hauswirtschaft erhält auf Basis aktueller gesellschaftspolitischer Diskussionen zum Thema Nachhaltigkeit eine ganz neue Relevanz. Sollte es gelingen, mittelfristig die hauswirtschaftliche Bildung und Berufsbildung in Richtung nachhaltige Entwicklung zu transformieren, könnten hauswirtschaftliche Fachkräfte durch professionelles nachhaltiges Handeln in vielen gesellschaftlichen Dimensionen einen ungemeinen sozialen, ökologischen und ökonomischen Einfluss nehmen.

Die der Hauswirtschaft zustehende gesellschaftspolitische Wertschätzung könnte auf diese Weise erreicht werden und damit allen hauswirtschaftlichen Bereichen neue Attraktivität verleihen.

„Bildung ist die mächtigste Waffe, um die Welt zu verändern“. Dem Zitat von Nelson Mandela gemäß könnte hauswirtschaftliche Bildung und Berufsbildung einen anerkannten Beitrag zu einer nachhaltigen Welt liefern.

\section{Literatur}

Hochschule für Agrar- und Umweltpädagogik (2018). Green Pedagogy. From theoretical basics to practical sustainable learning activities. Hochschule für Agrarund Umweltpädagogik, Vienna.

http://agrarumweltpaedagogik.ac.at/cms/upload/pdf/2018/

Hochschule/HAUP_GreenPedagogy_E_K2_2.pdf

Lave, J. (1988). Cognition in Practice. Mind, mathematics, and culture in everyday life. Cambridge, UK: Cambridge University Press.

https://doi.org/10.1017/CBO9780511609268

ProfESus Handbuch.

https://profesus.eu/fileadmin/user_upload/

Outputs/German/ProfESus_Handbook_de.pdf

ProfESus Newsletter | AUGUST 2018.

https://profesus.eu/fileadmin/user_upload/

Outputs/German/ProfESus_NL3_de.pdf

Sharples, M., Adams, A., Alozie, N., Ferguson, R., FitzGerald, E., Gaved, M. McAndrew, P., Means, B., Remold, J., Rienties, B., Roschelle, J., Vogt, K., Whitelock, D. \& Yarnall, L. (2015). Innovating Pedagogy 2015. Open University Innovation Report 4. Milton Keynes: The Open University.

http://iet.open.ac.uk/file/innovating_pedagogy_2015.pdf 


\section{Hauswirtschaftliche (Berufs-)Bildung}

Stanszus, L., Fischer, D., Boehme, T., Frank, P., Fritzsche, J., Geiger, S., Harfensteller, J., Grossman, P. \& Schrader, U. (2017). Education for Sustainable Consumption through Mindfulness Training: Development of a Consumption-Specific Intervention. Journal of Teacher Education for Sustainability, 19(1), 5-21. https://doi.org/10.1515/jtes-2017-0001

UNESCO (2015). Rethinking Education? Towards a global common good. UNESCO publishing 2015.

UNECE (2011). Learning for the future. Competences in Education for Sustainable Development.

http://unece.org/fileadmin/DAM/env/esd/

ESD_Publications/Competences_Publication.pdf

United Nations Environment Programme - UNEP (2010). HERE and NOW! Education for Sustainable Consumption Recommendations and Guidelines. A publication from the United Nations Environment Programme and the Marrakech Task Force on Education for Sustainable Consumption led by Italy in collaboration with the United Nations Decade on Education for Sustainable Development and the Hedmark University College in Norway. http://unep.fr/scp/marrakech/taskforces/pdf/H\&NMay2010.pdf

United Nations (o. J.). Sustainable Development Goals. $\mathrm{http} / / /$ sustainabledevelopment.un.org/sdgs

Weinert, F.E. (2001). Concept of competence: A conceptual clarification. In D.S. Rychen \& L.H. Salganik (eds.), (2001), Defining and selecting key competencies. (S. 45-65). Ashland, OH: Hogrefe \& Huber Publishers.

\section{Verfasserinnen}

Prof. ${ }^{\text {in }}$ Ing. ${ }^{\text {in }}$ Mag. ${ }^{\text {a }}$ Johanna Michenthaler, BEd

Hochschule für Agrar- und Umweltpädagogik Wien

Angermayergasse 1

A-1130 Wien

E-Mail: johanna.michenthaler@agrarumweltpaedagogik.ac.at

Dipl. troph. ${ }^{\text {in }}$ Anne von Laufenberg Beermann

Internationaler Verband für Hauswirtschaft

Kaiser-Friedrich-Straße 13

D-53113 Bonn

E-Mail: info@vlb-projektmanagement.de 